\title{
El aporte de la comunicación social a los proyectos de rehabilitación de redes de agua y saneamiento: el caso del Proyecto Lote 3 de Sedapal
}

Recibido: 23 de enero de 2019

Aceptado: 30 de abril de 2019

Publicado: 24 de junio de 2019
Wilfredo Cornejo Alva dcornejoa@pucp.pe

Resumen: El presente artículo reseña la implementación de estrategias de comunicación desarrolladas en el Proyecto de Rehabilitación de Redes de Agua Potable y Alcantarillado Lote 3, ejecutado entre los años 2013 y 2014 en el distrito de Comas, al norte de Lima (Perú) y perteneciente al Servicio de Agua Potable y Alcantarillado de Lima (SEDAPAL). Como resultado, pudo observarse que la implementación de un diagnóstico de percepciones, el plan de comunicación y los productos de comunicación y educación permitieron facilitar el diseño de mensajes acordes al contexto del proyecto, tomando en consideración los discursos de los usuarios en contra y a favor del proyecto, así como los objetivos buscados por la empresa ejecutora.

Palabras clave: Agua, comunicación, intervención social, comunicación para el desarrollo, relaciones comunitarias, saneamiento.

Abstract: This paper focus on implementation of communication strategies developed in the Rehabilitation of Potable Water and Sewerage Network Lote 3 project, executed between 2013 and 2014 in the district of Comas, north of Lima-Peru, and belonging to the Servicio de Agua Potable y Alcantarillado de Lima (SEDAPAL), company responsible for water and sewage in Lima. As a result, it could be observed that the implementation of a diagnosis of perceptions, the communication plan and the communication and education products allowed to facilitate the design of messages according to the context of the project, taking into account the speeches of the users against and in favor of the project, as well as the objectives sought by the executing company.

Key words: Water, Communication, Social Intervention, Communication for Development, Community Relations, Sanitation. 


\section{Introducción}

Los proyectos de rehabilitación de redes de agua y saneamiento traen muchos beneficios para la población: la reducción del riesgo de aniegos que pueden afectar las viviendas de los usuarios; la continuidad y mejora del servicio, manteniendo o aumentando la presión del agua o reduciendo malos olores producto del alcantarillado en inadecuadas condiciones; la posibilidad de ampliar las redes para quienes aún esperan tener agua y desagüe en sus domicilios en zonas geográficas más altas como cerros, por ejemplo, y con ello ofrecer mejoras en la salud y más oportunidades para el desarrollo de la población.

\subsection{Lima y los proyectos de rehabilitación}

Lima es una ciudad desértica, como toda la costa peruana. Durante la Conquista se escogió la configuración geográfica de la actual capital del Perú en base, entre otros elementos, a contar con un río (el Rímac) que les provea de agua dulce y acceso directo al mar. En su momento, el Rímac fue suficiente para abastecer a la ciudad. Sin embargo, hoy cuenta con más de 9 millones de habitantes (INEI, 2017) quienes hacen uso de redes de agua y alcantarillado que, aunque no llegan a cubrir a toda la ciudad, requieren de rehabilitación.

La rehabilitación de redes consiste en una serie de actividades de ingeniería, a fin de restablecer el correcto funcionamiento de los sistemas de agua y alcantarillado (tuberías, reservorios, cámaras, bombas, entre otros). La rehabilitación es sustancial para que la población siga recibiendo estos servicios. No obstante, estos proyectos generan numerosas incomodidades y riesgos para los ciudadanos: aperturas de zanjas, ruido, polvo, presencia de personas extrañas, material de construcción en sus vías, afectaciones a veredas y jardines, imposibilidad del uso de cocheras durante las obras, entre otros.

Uno de esos proyectos de rehabilitación desarrollados en la ciudad capital fueron las obras de rehabilitación de redes Secundarias de agua potable y alcantarillado lote 3 (o proyecto "Lote 3", como se le llamará en adelante), gestionado por el Servicio de Agua Potable y Alcantarillado de Lima (SEDAPAL) y ejecutado por la empresa constructora Consorcio Lima Norte (CLN).

A fin de procesar los riesgos de la obra y otros posibles conflictos, el CLN contó con un equipo de intervención social (EIS) conformado por 8 profesionales, entre ellos un comunicador social. El EIS era responsable de "mantener una permanente comunicación y una estrecha coordinación con la población" con el fin de "prever y evitar demoras y retrasos en obra" (SEDAPAL, 2011a). Es en ese contexto donde se incorpora la labor del comunicador social, el cual contribuye con herramientas estratégicas para los fines ya mencionados.

El Lote 3 fue uno de los primeros proyectos de rehabilitación de gran envergadura en Lima, beneficiando a 22857 usuarios (SEDAPAL, 2011b) del distrito de Comas, al norte de la ciudad. A diferencia de las obras de ampliación de redes, que son bien recibidas por la población, debido a que estas traen el servicio de agua por primera vez, este proyecto de rehabilitación generó un alto rechazo por la cantidad de impactos que generó, la experiencia con otros proyectos abandonados o que no tuvieron los beneficios esperados por los usuarios. 


\subsection{Las estrategias de comunicación del proyecto}

En los proyectos de saneamiento era común contar con un personal del equipo social denominado "capacitador", encargado de desarrollar productos netamente educativos para talleres comunitarios. Sin embargo, en esta oportunidad se desarrollaron métodos de investigación y de planificación en comunicación con el objetivo de difundir y posicionar de forma más estratégica el proyecto. Se buscaba una comunicación que fomente el diálogo (Fraser \& Restrepo-Estrada, 2008, p. 792), que promueva un cambio y para eso era necesario conocer a los usuarios.

El público objetivo primario, a quienes se dirigieron las estrategias de comunicación, lo constituyeron pobladores usuarios de los servicios, grandes negocios, comerciantes que vieron afectados parcialmente sus lugares de trabajo, miembros de centros de salud que podrían verse impactados, organizaciones sociales, religiosas y similares (CLN, 2012). Las herramientas de comunicación más im portantes desarrolladas durante el proyecto fueron el diagnóstico de percepciones sobre los servicios de agua potable y alcantarillado, el plan de comunicaciones y los productos de comunicación.

\section{Objetivo}

El objetivo de la presente investigación es analizar las lecciones aprendidas en la labor del comunicador social del equipo de intervención social del proyecto de rehabilitación de redes de agua potable y alcantarillado (Lote 3), a partir de las herramientas de comunicación desarrolladas: un estudio de percepciones sobre el proyecto Lote 3, el plan de comunicaciones y los productos de comunicación.

\section{Metodología}

Para la presente investigación se desarrollaron tres procesos: a) la revisión de fuentes documentales para la reconstrucción de la experiencia; b) la revisión de ejes temáticos vinculados y c) entrevistas en profundidad al coordinador del equipo de intervención social y al supervisor social del proyecto Lote 3 .

La revisión de fuentes de la experiencia consistió en el análisis de los documentos generales del proyecto, como los términos de referencia, las especificaciones técnicas, el plan de intervención social, el plan de contingencias, así como los informes mensuales del equipo de intervención social.

Respecto a la revisión de ejes temáticos, esta consistió en la búsqueda bibliográfica vinculada con las temáticas como agua, relaciones comunitarias y comunicación para el desarrollo, con el fin de dilucidar lo que propone la academia. Finalmente, otro elemento de análisis lo constituyeron las entrevistas en profundidad que se realizaron tanto al jefe del equipo de intervención social del proyecto y al supervisor social de la obra. 


\section{Resultados}

Se pudo evidenciar que el estudio de percepciones realizado se convirtió en la columna vertebral de toda la estrategia y los productos de comunicación. Gracias a este esfuerzo se detectaron las posiciones de los usuarios frente al proyecto Lote 3 , algunas a favor y otras en contra, pero sobre todo las razones que justificaban esas opiniones a fin de poder interpelar sus premisas para convencerlos a favor del proyecto (Frenette \& Reyes, 2011, p. 38). Asimismo, se solicitó sobre los servicios de agua potable y alcantarillado en general, respecto de los trabajos que SEDAPAL previamente había realizado; los medidores de agua, que aunque no formaban parte del proyecto eran un tema sumamente delicado en algunas zonas del distrito por su mala reputación; y finalmente sobre el propio proyecto Lote 3 , si los usuarios tenían algún conocimiento sobre él.

Respecto al plan de comunicaciones, este se desarrolló con el apoyo del coordinador del EIS, además de la consulta con otros especialistas en comunicación con experiencia en relaciones comunitarias. Así, se prepararon mensajes que buscaban reducir la animadversión hacia el proyecto, aclarando algunos tópicos detectados en el estudio de percepciones. También se consideró la elaboración de numerosos productos de comunicación.

Se estableció como público primario a los usuarios, a quienes se les informaría de los beneficios de la obra, sobre los posibles impactos de obra y a los que se les pediría una mejora en el uso de sus instalaciones sanitarias (Acosta, 2014). Además, se tomó en cuenta los comerciantes y dueños de negocios que verían impactados sus ingresos, dado que las obras requerían el cierre de calles y la apertura de zanjas. Otro grupo a considerar fueron los centros de salud y centros educativos.

Los productos de comunicación desarrollados no fueron todos los planteados en el plan de comunicación. No obstante, se desarrollaron tres principales: un volante, un afiche y un tríptico del proyecto que consideraban los mensajes sugeridos en el plan. Respondiendo a sus términos de referencia, se prepararon adicionalmente seis rotafolios con temas para la capacitación de usuarios buscando, entre otros fines, la mejora de las prácticas en el uso del agua y reducir su gestión ineficiente en los domicilios por una cultura inadecuada (Aragón, 2012, p. 64). Para ello se realizaron consultas bibliográficas, verificación de datos, consultas y trabajo cercano con la supervisión social, a fin de brindar información confiable. También en este proceso se consideraron las conclusiones del estudio de percepciones.

\section{Discusión}

\subsection{El contexto}

El Equipo de Intervención Social (EIS) estuvo conformado por un equipo de nueve personas: un coordinador, un comunicador social y siete promotores sociales con las siguientes profesiones: dos sociólogos, tres trabajadores sociales, un educador y un comunicador 
social que realizó la labor de promotor, es decir, de contacto directo y atención en campo de cualquier inconveniente con la población.

La obra fue supervisada por la empresa Nippon Koei Lac, que para tal efecto contó con un sociólogo que tenía la función de verificar, observar y aprobar las actividades. La labor de este supervisor debía considerarse porque todos los procedimientos y materiales de comunicación desarrollados durante el proyecto requerían de su aprobación, por lo que siempre se requirió de un diálogo y gestión con el mencionado sociólogo.

Adicionalmente, SEDAPAL contaba en ese momento con un equipo social. Este equipo se encargaba de la verificación de las condiciones sociales de los proyectos de su cartera en todo Lima Metropolitana. También era una última instancia en casos de discrepancia entre la empresa supervisora y la empresa ejecutora. No obstante, su labor fue muy lejana y específica para determinadas ocasiones.

El Consorcio Lima Norte (CLN) no contó con una política propia que enmarque sus actividades en el aspecto social. No obstante, estuvo provisto de otros documentos que permitieron guiar su intervención social, los que incluían:

- Plan de contingencias del equipo de intervención social: precisaba los procedimientos a seguir ante determinados escenarios favorables o desfavorables sobre el proyecto.

- Especificaciones técnicas del proyecto: describía los procedimientos técnicos necesarios para el desarrollo del proyecto. Los procedimientos de ingeniería estaban muy bien detallados, sin embargo, los referidos a las relaciones con la comunidad eran muy reducidos, casi inexistentes.

- Guías gráficas de SEDAPAL: se contó con el manual de identidad de SEDAPAL, el cual ofrecía los lineamientos necesarios para el diseño de materiales de comunicación alineados a la imagen de la mencionada empresa cliente del CLN.

- Estudio de Impacto Ambiental (EIA): el correspondiente EIA del proyecto Lote 3 estaba incluido en otro de mayor alcance que abarcaba otros proyectos de rehabilitación e incluía cuatro distritos. Incluía información socioeconómica con datos sumamente generales pero que aportaron ciertos lineamientos de trabajo.

\subsection{Sobre el estudio de percepciones de los usuarios acerca de los servicios de agua potable y alcantarillado}

Al inicio del proyecto, el EIS no contó con un diagnóstico social focalizado que incluyese información detallada sobre la opinión de la población sobre el Lote 3. Se desarrolló un rápido levantamiento de información sobre los actores presentes en la zona de trabajo y si estaban de acuerdo o no con la obra, pero de forma muy superficial. El EIA describió algunos aspectos demográficos, pero a un nivel macro, por lo que no era de relevancia.

Ante ello, el comunicador del proyecto propuso al coordinador del EIS la realización de un estudio de percepciones a fin de conocer las razones del apoyo o rechazo que pudiese existir frente al proyecto, como proponen los nuevos modelos de comunicación para el 
desarrollo (Alfaro, 2006, p. 79). Gracias a dicho estudio, fue posible la segmentación de los públicos con el fin de preparar productos, mensajes y discursos, con la finalidad de promover o gestionar la aceptación del proyecto entre los actores presentes.

Se realizaron entrevistas a 30 usuarios representativos (dirigentes usuarios de agua potable, dueños de negocios y otros líderes). Los promotores sociales, con zonas de trabajo determinadas, se encargaron de realizar este primer acercamiento. No obstante, debido a la demanda en sus labores (especialmente de atención de los primeros conflictos en sus lugares de trabajo dado que los trabajos operativos ya se habían iniciado), el diagnóstico se realizó de manera ágil. A pesar de la situación descrita, la información recogida fue sumamente valiosa.

En el estudio de percepciones se incluyeron cuatro temas que cubriesen aspectos de lo más amplio hasta lo más específico. Así, se consultó sobre: a) los servicios de agua potable y alcantarillado en general, b) las obras de SEDAPAL, c) los medidores de consumo de agua potable y d) el proyecto Lote 3.

Se preguntó sobre los servicios de agua potable y alcantarillado en general para obtener la primera impresión que tenían los usuarios acerca de sus servicios, a fin de detectar también algún insight que nos permitiese direccionar los mensajes o la estrategia en general. Vale mencionar que esto nos permitiría un cierto acercamiento a la cultura del agua de los usuarios, entendida esta como el conocimiento y las prácticas en su uso y cuidado (Aquafondo, 2014).

SEDAPAL, como parte de las acciones de mantenimiento de las redes, siempre desarrolla acciones correctivas con el fin de continuar dando el servicio. De por sí, es conocido por la población el bajo rendimiento de las atenciones de la empresa al llamado de sus clientes. Como se iba a iniciar un proyecto amplio de rehabilitación, por ello era necesario conocer qué percepción tenían los usuarios de la zona acerca de esos trabajos.

Asimismo, se incluyeron algunas interrogantes sobre los medidores de agua. Esto se dio porque, realizando la consulta con personal social con mayor experiencia en trabajos de sistemas de agua y alcantarillado, pudo evidenciarse que la instalación de medidores de consumo era un tema sumamente polémico para los usuarios, debido a que lo asociaban con facturaciones altas del servicio. Por esta razón también se incluyó en el estudio, a fin de determinar si eso se replicaba entre los actores de la zona. Finalmente, luego de tener todo este marco, recién se pasaba a preguntar a los usuarios acerca del proyecto Lote 3 , si recordaban el haber tenido alguna información previa ${ }^{1}$.

Entre las interrogantes incluidas (tabla 1) se formularon las siguientes: ¿Ha tenido algún inconveniente con el servicio de agua/alcantarillado en su barrio?, ¿qué opina sobre los trabajos que realiza SEDAPAL en las calles?, ¿conoce cuáles son los proyectos que SEDAPAL ha venido realizando a fin de mejorar su servicio?, ¿qué opina sobre los medidores

1 Vale decir que como todo proyecto de inversión pública, en la etapa de preinversión debía haberse realizado la difusión del proyecto, Sin embargo, esas acciones se habían desarrollado algunos años atrás, por lo que probablemente la población no recordara los mensajes de aquellas campañas. 
de agua?, ¿ha escuchado hablar de algún proyecto para el cambio de las redes principales de agua y alcantarillado y las conexiones domiciliarias en esta parte de Comas? Luego de las entrevistas, se reunió a los promotores sociales para que compartieran cuáles habían sido las respuestas de los entrevistados y los comentarios acerca de la aplicación de la prueba.

Adicionalmente se buscó estudios de satisfacción sobre los servicios de SEDAPAL. El único encontrado al momento del desarrollo del proyecto fue una encuesta bianual (SEDAPAL, 2010) pero que no incluía a los trabajos de campo de la empresa, sino sólo abarcaba la atención de sus oficinas de atención al cliente. Así, con el apoyo de los promotores sociales de campo, se pudo obtener información valiosa de primera mano, de interés incluso para el cliente (SEDAPAL) y, sobre todo, que permitió mejorar la propuesta discursiva para el equipo.

Tabla 1: Cuestionario del Estudio de percepciones sobre el Lote 3.

\begin{tabular}{|l|}
\hline Sobre los servicios de agua potable y alcantarillado en general \\
\hline ¿Ha tenido algún inconveniente con el servicio de agua en su barrio? \\
\hline ¿Ha tenido algún inconveniente con el servicio de desagüe (alcantarillado) en su barrio? \\
\hline Sobre las obras de SEDAPAL en general \\
\hline ¿Qué opina sobre los trabajos que realiza SEDAPAL en las calles? \\
\hline ¿Conoce cuáles son los proyectos que SEDAPAL ha venido realizando a fin de mejorar su servicio? \\
\hline Sobre los medidores de agua \\
\hline ¿Qué opina sobre los medidores de agua? \\
\hline ¿Estaría de acuerdo con que se le instale un medidor de agua en su domicilio?, ¿por qué? \\
\hline Sobre el proyecto Lote 3 \\
\hline $\begin{array}{l}\text { SEDAPAL realizará un gran proyecto que permitirá aumentar el servicio de agua a } 24 \text { horas diarias.La obra } \\
\text { incluirá trabajo con zanja y, en algunas zonas, tecnología sin zanjas, para disminuir los impactos de las } \\
\text { obras. ¿Qué esperaría de estos trabajos de SEDAPAL? }\end{array}$ \\
\hline
\end{tabular}

Fuente: Cornejo (2015, p. 52).

Con respecto a los servicios de agua potable y alcantarillado, se detectó que había zonas en las que se tenía agua las 24 horas y otras en las que el servicio era por horas. Los primeros reclamaban que padecían de cortes intempestivos (no programados ni informados), mientras que los segundos exigían la ampliación del horario de abastecimiento y reclamaban por sus altos costos. Adicionalmente, estos últimos mencionaron que el aire que eliminaban las tuberías cada vez que llegaba el agua generaría problemas con eventuales medidores de consumo, porque estos registrarían el aire como si fuera agua y eso aumentaría irregularmente la facturación mensual.

Sobre el alcantarillado, los entrevistados manifestaron quejas por atoros. Los usuarios de las zonas bajas y consolidadas (con calles pavimentadas y veredas) responsabilizaban a los usuarios de las zonas altas ubicados en los cerros, pero nunca mencionaban la posibilidad que ellos mismos tuvieran algo que ver con el inconveniente.

Consultados sobre los trabajos realizados por SEDAPAL, los usuarios mencionaron que estos eran superficiales, que solucionaban los inconvenientes únicamente de forma 
temporal y no definitiva y que además los atoros de alcantarillado, por ejemplo, volvían a ocurrir poco tiempo después de los arreglos. Las intervenciones de otras empresas contratistas fueron criticadas, además de su pobre efectividad, por los hoyos que dejaban en su zona de trabajo sin tapar o los materiales olvidados que generaban luego accidentes con daños materiales o personales especialmente en los niños que jugaban en la zona. También comentaron que los plazos de ejecución nunca se cumplían y que la señalización de seguridad de las obras era deficiente o inexistente.

Los usuarios, que vivían cerca de zonas donde SEDAPAL había realizado trabajos de mantenimiento, sabían que estos eran necesarios para que continúen recibiendo el servicio. Los que no veían estas intervenciones no entendían su necesidad a pesar que eran también beneficiarios de las mismas. Todo esto producía una enorme desconfianza entre los usuarios respecto a cualquier trabajo que pudiese realizar SEDAPAL.

Respecto al proyecto Lote 3, se evidenció que la mayoría de los dirigentes entrevistados desconocía el proyecto por completo y no comprendía su necesidad ni su naturaleza. Al mencionarles que esta obra era parte de un proceso por el cual ellos lograrían tener agua las 24 horas (en el caso de aquellos que no tenían ese beneficio) para la mayoría les era indiferente o no le daban credibilidad a esa información, dado que ya habían recibido esa promesa anteriormente en reiteradas ocasiones y que esta no había sido cumplida. De otro lado, casi todos desconocían las importantes obras que SEDAPAL había realizado para traer agua desde la sierra de Lima o la región de Junín. Otros comentaban que la obra representaba simplemente un motivo para "romper la pista", hecho que no debía pasar desapercibido, ya que en numerosos barrios los propios vecinos habían financiado la pavimentación de sus calles, las que ahora tendrían que verse afectadas por los trabajos.

Finalmente, se consultó a los usuarios sobre la instalación de medidores de agua. Es importante señalar que el proyecto Lote 3 no contemplaba la instalación de medidores; no obstante, fácilmente la población podría interpretar que el proyecto incluía este componente al vincularlo con los servicios de agua potable. Además, como ya fue mencionado, los medidores eran un tema sumamente polémico por lo que era necesario medir la percepción al respecto.

Para los usuarios que ya contaban con el medidor su instalación les parecía satisfactoria porque con este disminuyó el consumo y el pago por el servicio. Sin embargo, otro grupo mencionaba que este instrumento aumentaría el costo del servicio argumentando que los marcadores (similares a las agujas de un reloj) giraban solas, de forma automática. Este hecho, no obstante, puede darse por fugas no visibles en los domicilios que hacen que el flujo de agua continúe, aunque se tengan todas las llaves cerradas. Los promotores sociales, sin embargo, encontraron también un pequeño grupo de usuarios que sí esperaba los medidores y que incluso personal de SEDAPAL les había ofrecido su instalación pero que nunca se había concretado.

Respecto al estudio de SEDAPAL sobre satisfacción en la atención a sus clientes, este fue limitado para la presente investigación (tabla 2), debido a que las consultas realizadas se referían únicamente a los servicios en las oficinas de atención al público, sin considerar sus trabajos en los barrios o en las calles de sus usuarios, indispensables para que brinden sus servicios. 
Tabla 2: Resumen de resultados del estudio de percepciones.

\section{Percepciones sobre los servicios de agua potable y alcantarillado en general}

Usuarios con servicio de agua las 24 horas:

- Se quejan de las suspensiones del servicio.

- No son informados adecuadamente de las suspensiones del servicio.

- Programaciones de obra incumplidas.

- No se conocen las razones de los cortes.

- Quejas cuando hay roturas de redes.

- "SEDAPAL no llega", "se demoran" (usuario se siente ignorado).

Usuarios con servicio de agua por horas:

- Abastecimiento por horas e insuficiente.

- Horarios inoportunos: "Llega cuando salgo a trabajar".

- Alto costo por un servicio limitado.

- "Las tuberías botan aire", lo que genera desconfianza en los medidores.

Sobre el servicio de alcantarillado:

- Quejas por atoros.

- Viviendas de zonas bajas responsabilizan a los vecinos de las zonas altas por sus malas prácticas.

- Las inundaciones generan paredes húmedas y debilitamiento.

- Las soluciones de SEDAPAL son superficiales.

- El abandono de las obras produce la aparición de roedores.

\section{Sobre las obras de SEDAPAL en general}

- El compromiso de dejar la zona en iguales o mejores condiciones es hoy insuficiente.

- Comentarios negativos se basan en experiencias anteriores: abandonos de obras, incumplimientos de plazos, promesas incumplidas de contratistas.

- Cierres de obra deficientes producen accidentes y daños personales y materiales.

- Usuarios alejados de las zonas de obra saben de su existencia, pero no de sus razones ni beneficios.

\section{Sobre los medidores de agua}

Usuarios sin medidores:

- "El medidor es técnicamente deficiente".

- "Genera sólo beneficios para SEDAPAL".

- "Luchamos por no tenerlos".

Usuarios con medidores:

- Satisfechos.

- Registra de forma automática (gira solo, pero debido a fugas no visibles).

Usuarios que esperan medidores:

- Los ofrecieron en proyectos anteriores.

- Los han solicitado.

\section{Sobre el proyecto Lote 3}

Respecto a la propuesta de 24 horas de agua gracias al proyecto:

- Es bien recibida.

- Genera indiferencia e incredulidad (promesas anteriores de SEDAPAL y otros contratistas).

- Desconocimiento de grandes obras de SEDAPAL.

- Críticas de los usuarios: "Otra vez van a romper la pista", que en algunos casos era pavimentada con recursos económicos de los vecinos.

Fuente: Cornejo (2015, p. 54). 


\subsection{El diseño del plan de comunicación}

El estudio de percepciones brindó grandes luces sobre el ideario de la población beneficiaria respecto al proyecto Lote 3, así como a otras temáticas vinculadas (las obras previas de SEDAPAL, la calidad de su servicio y los medidores de agua en general). Esta información fue sumamente valiosa para poder preparar el plan de comunicación del proyecto.

En un principio se planteó desarrollar un enfoque de comunicación corporativa; sin embargo, dada la información que arrojó el estudio de percepciones y, adicionalmente, a las experiencias previas del coordinador del Equipo de Intervención Social (EIS) en proyectos previos de saneamiento, el plan cambió de enfoque hacia uno de relaciones comunitarias. Esto permitió, además, un actuar meditado, planificado y justificado (Moliner, 1998, p. 48).

El plan incluyó una descripción de la situación en que se encontraba la zona misma del proyecto y los impactos que esta iba a generar: apertura de zanjas, cierre de calles, presencia de personas extrañas, riesgos, ruidos, polvo, material de construcción y maquinaria pesada en las calles. También se detallaron los servicios que podrían verse afectados a consecuencia del proyecto: electricidad, telefonía, cable, internet, entre otros. Socialmente pudo detectarse del vínculo cercano existente entre los vecinos usuarios. En algunos casos, incluso, los hijos de las familias más antiguas vivían ya habiendo formado sus propios hogares muy cerca e incluso construyendo departamentos en los segundos y terceros pisos de las viviendas de sus progenitores.

Con respecto a la definición del público objetivo, se priorizaron aquellos grupos de actores que se verían más afectados por el proyecto: los que vivían en la propia zona de influencia directa, los dueños de negocios, así como comerciantes de la zona. También se consideraron a los centros de salud públicos y privados. Como público secundario se consideraron a las entidades educativas, así como a los representantes locales; si bien habían perdido vigencia, en un eventual conflicto suelen reactivarse.

Ante la diversidad de ideas y posiciones respecto al proyecto Lote 3, se decidió crear un conjunto de mensajes claves (tabla 3) para los diferentes y probables escenarios que enfrentarían los promotores sociales en campo, considerando la cultura y el contexto de los usuarios.

Tabla 3: Mensajes clave indicados en el plan de comunicaciones.

\begin{tabular}{|ll|}
\hline Sobre el cliente y el contratista: \\
\hline - & SEDAPAL trabajando para ti. \\
\hline Sobre el propósito e importancia del proyecto: \\
\hline - & Con la rehabilitación de redes de Agua y Alcantarillado ahora sí tendrás agua las 24 horas. \\
- & SEDAPAL: hacemos grandes obras e inversiones para la mejora de tu calidad de vida. \\
- & SEDAPAL ha ejecutado grandes megaproyectos para que tengas agua las 24 horas. \\
- & Ahora verás los resultados con el proyecto Lote 3. \\
& SEDAPAL utilizará tecnología para cambiar redes de agua y alcantarillado sin zanjas. Todo para darte un \\
\hline & mejor servicio. \\
\hline
\end{tabular}




\section{Sobre las obras de trabajo:}

- Trabajamos para un mejor servicio de agua y alcantarillado.

- Pronto visitaremos tu domicilio para ofrecerte la debida información de los trabajos y de las obras.

- Disculpe la molestia, estamos trabajando para que tenga un mejor servicio de agua y alcantarillado. Gracias por su comprensión.

- Su comprensión es importante para alcanzar un mejor servicio de agua y alcantarillado.

- ¿Deseas más información o alcanzar alguna sugerencia? Estamos atentos a escucharle.

Seguridad y prevención por las obras:

- Lee y respeta siempre las señales de seguridad. Así evitaras accidentes.

- No ingreses al área de trabajo por tu seguridad. Te librarás de los accidentes.

- Informa a tu familia sobre las señales de seguridad y haz que las respeten.

Valoración del agua potable:

- Gota a gota, el agua se agota.

- El agua que se cuida, es agua que sí te alcanza.

- Cuida el agua para que más usuarios la disfruten.

Mejora de las instalaciones de agua y alcantarillado:

- Manteniendo mis tuberías, ayudo a mi economía.

- Con las fugas perdemos todos. Revisa tus instalaciones y ¡arregla las fugas de una vez!

- No elimines basura al desagüe. Así evitaremos los atoros.

- Cuidando las tuberías de agua y desagüe; así aseguro mi salud y la de mi familia.

Micromedición:

- Con los medidores de SEDAPAL, en agua, yo sí pago lo justo.

- Con el medidor mido mi consumo y, encima, ¡ahorro!

- $\quad$ El reloj mide tu tiempo. El medidor, tu agua, para que sí pagues lo justo.

- $\quad$ Ni aire, ni nada. El medidor sólo mide el paso del agua

Fuente: CCLN (2012).

Con referencia a los productos de comunicación a utilizarse, se plantearon numerosas propuestas clasificadas en cuatro grupos: a) impresos personales, que se referían al diseño de volantes, folletos o cartillas para lectura individual; b) impresos públicos, en los que se incluían afiches, banderolas, letreros de obra y anuncios periodísticos de cortes de agua; $c$ ) audiovisuales y $d$ ) spots radiales.

Adicionalmente al trabajo en conjunto (desarrollado con el coordinador del EIS del proyecto) para el desarrollo del plan se consideró buscar a otros comunicadores con experiencia en estrategias de relaciones comunitarias, debido a la naturaleza particular de este tipo de intervenciones. Por ejemplo, el contacto con el público objetivo (los usuarios de los servicios de agua y alcantarillado) debía ser continuo y de mediano plazo, dado que las obras así lo requerían.

Otro aspecto a considerar era el uso de medios de comunicación locales y el desarrollo de nuestras propias herramientas de comunicación. El ofrecer un mensaje en un medio de comunicación masivo hubiese conllevado también el riesgo de que aparecieran caudillos o líderes negativos buscando sacar provecho de la intervención y de la percepción negativa de la población. Por ese motivo, era conveniente trabajar de forma muy localizada. También se consideró que, dentro del público objetivo, la mayoría era público femenino que se encontraba en casa realizando 
labores de amas de casa al momento que el promotor social llegaba a su domicilio. Esto permitió enfocar los esfuerzos comunicativos en centrar los mensajes vinculados al rol que ellas desarrollaban: la administración del hogar y el cuidado de la familia.

Los proyectos de construcción cuentan también con planes de mitigación para los impactos de la obra. De hecho, las contratistas cuentan con equipos de salud, seguridad ocupacional y medio ambiente (SSOMA). Así, se trabajaron algunos mensajes con ese equipo del consorcio, vinculados a prevenir el ingreso a las zonas de trabajo.

Aunque la obra tenía como beneficios la continuación y especialmente la mejora de los servicios, esta no era inmediata. Ante los usuarios, la primera consecuencia de la obra serían no sus beneficios, sino sus impactos, los que generarían una serie de incomodidades sumadas a las ya identificadas en el estudio de percepciones. En ese sentido, se decidió tener como público objetivo primario a los usuarios dado que estos (frente a impactos no gestionados adecuadamente) podrían generar un conflicto.

Además, se tomó en cuenta a los dueños de negocios y comerciantes del lugar, porque variaría la circulación de vehículos y de clientela por sus lugares de trabajo, afectando sus ingresos, así como el transporte de sus productos, materias primas y similares. A este grupo se le explicó claramente la duración de los trabajos y se coordinó internamente el brindarles las facilidades técnicas para reducir estos daños, como la instalación de pequeños puentes para acceso peatonal y el control cercano del cierre de zanjas. Se consideró a las entidades de salud como otro público especial dado el servicio sensible que brindan a la población. Finalmente, otras organizaciones sociales y educativas fueron consideradas de manera secundaria para replicar los mensajes.

Los mensajes clave incluyeron, en principio, los básicos solicitados por SEDAPAL que permitan identificar a la empresa contratista como ejecutora de proyectos autorizados ("Consorcio Lima Norte: empresa ejecutora trabajando para SEDAPAL"). Pero este no era suficiente. Era necesario implementar otros discursos, especialmente por la alta desconfianza detectada en el estudio de percepciones.

Se implementaron mensajes adiciones vinculados a los beneficios del proyecto Lote 3, vinculados a la disposición futura de ofrecer 24 horas de agua potable. En otros mensajes se mencionaba el hecho de que las incomodidades eran necesarias para poder continuar recibiendo el servicio ("En SEDAPAL toda obra que hacemos es para ofrecerte un mejor servicio"). También se mencionaba la inversión que realizaba la empresa cliente para beneficio de los usuarios ("SEDAPAL: hacemos grandes obras e inversiones para la mejora de tu calidad de vida", "SEDAPAL ha ejecutado grandes megaproyectos para que tengas agua las 24 horas. Ahora verás los resultados con el proyecto Lote 3").

Una de las novedades de este proyecto era el sistema de cambio de tuberías sin zanjas, un procedimiento que a través de martillos neumáticos evitaría la excavación de zanjas, hecho que también se expresó en otro de los mensajes clave ("SEDAPAL utilizará tecnología para cambiar redes de agua y alcantarillado sin zanjas. Todo para darte un mejor servicio"). 
Dada la desconfianza mencionada por los usuarios, se buscó establecer mensajes que mostrasen la labor de SEDAPAL por la mejora de las redes y del servicio para sus clientes, así como la cercanía que se deseaba establecer entre la empresa ejecutora y los usuarios ("Trabajamos para un mejor servicio de agua y alcantarillado", "Pronto visitaremos tu domicilio para ofrecerte la debida información de los trabajos y de las obras", "Disculpe la molestia, estamos trabajando para que tenga un mejor servicio de agua y alcantarillado. Gracias por su comprensión"). También se buscaba manifestar la apertura como empresa ejecutora para escuchar al usuario, a fin de que reporte alguna anomalía (“¿Deseas más información o alcanzar alguna sugerencia? Estamos atentos a escucharle"). Esta estrategia complementó la estrategia del coordinador social y el equipo de promotores sociales de realizar un acompañamiento permanente en campo, lo que facilitaba la atención rápida de incidencias.

Una zanja de metro y medio de profundidad siempre representará un riesgo para cualquier ciudadano, más aún si se cuenta con niños que podría jugar peligrosamente en la zona o incluso para las mascotas del lugar. Por ello se prepararon mensajes que reforzaran la labor del equipo de SSOMA y que invocaban a que los usuarios comuniquen a sus familias sobre los peligros en caso de no seguir las señalizaciones correspondientes ("Lee y respeta siempre las señales de seguridad. Así evitarás accidentes", "No ingreses al área de trabajo por tu seguridad. Te librarás de los accidentes", "Informa a tu familia sobre las señales de seguridad y haz que las respeten").

Pero el EIS no se dedicaba sólo a la prevención y gestión de conflictos. También tenían como responsabilidad la promoción de un buen uso del agua potable y del alcantarillado. Así, se consideró un mensaje solicitado por SEDAPAL y utilizado ya desde mucho tiempo atrás ("Gota a gota, el agua se agota"), así como de otros que también solicitaban el cuidado del recurso ("El agua que se cuida, es agua que sí te alcanza", "Cuida el agua para que más usuarios la disfruten").

Otra responsabilidad que debía recordar a los usuarios era la de arreglar y conservar sus instalaciones. Este hecho es fundamental porque permite que, por un lado, no se pierda agua potable considerando que Lima es una ciudad ubicada en un desierto y porque toda pérdida reduce la presión, reduciendo la posibilidad de que el recurso llegue a zonas altas como segundos pisos o asentamientos humanos en los cerros ("Manteniendo mis tuberías, ayudo a mi economía", "Con las fugas perdemos todos. Revisa tus instalaciones y jarregla las fugas de una vez!").

El rol de los usuarios en el cuidado del desagüe o alcantarillado también es fundamental. Los atoros se deben directa y únicamente al hecho de que de forma irresponsable algunos eliminan desechos sólidos por los sistemas. Al acumularse, esto produce la obstrucción y el colapso de los sistemas. Por ello, se incluyeron mensajes que procuraban exhortar a los usuarios a no utilizar los desagües como basureros ("No elimines basura al desagüe. Así evitaremos los atoros", "Cuidando las tuberías de agua y desagüe; así aseguro mi salud y la de mi familia"). 
Finalmente, respecto a los medidores de agua, se buscó informar a los usuarios de que, al margen de este dispositivo, el consumo de agua responde a los usuarios. Se rescató también el hecho de que algunos usuarios mencionasen que luego de contar con el medidor sus facturaciones se habían reducido. Entonces, se construyeron mensajes que consideren ambos hechos ("Con los medidores de SEDAPAL, en agua, yo sí pago lo justo", "Con el medidor mido mi consumo y, encima, jahorro!", "El reloj mide tu tiempo. El medidor, tu agua, para que sí pagues lo justo", "Ni aire, ni nada. El medidor sólo mide el paso del agua"). Como puede observarse, se desarrolló un conjunto de mensajes acordes con lo detectado en el estudio de percepciones más lo sugerido por comunicadores con experiencia en relaciones comunitarias, así como por lo expresado por el coordinador y los promotores del EIS.

\subsection{Los productos de comunicación implementados}

No se llegaron a desarrollar todos los productos de comunicación propuestos en el plan. Sin embargo, a medida que el proyecto se desarrollaba, se fueron implementando las herramientas que se consideraban adecuadas y necesarias para una determinada situación. Los tres productos de comunicación impresos más utilizados fueron el volante informativo de obra, el afiche de obra y el tríptico informativo.

Adicionalmente, se desarrollaron materiales de capacitación solicitados por los términos de referencia del proyecto (SEDAPAL, 2011b), Estos materiales consistían en rotafolios y folletos que explicaban temáticas vinculadas al proyecto. Se trataba de dos grandes talleres, el primero se describía los sistemas de agua potable y alcantarillado, las bondades del proyecto, la micromedición y el uso racional del agua potable y del alcantarillado. El segundo taller incluía temas de educación sanitaria, el uso adecuado de los sistemas de evacuación de desagües y la importancia de la mejora de las instalaciones interiores.

Debido a la gran cantidad de información, se decidió reorganizar estas temáticas y se acordó (con aprobación de la supervisión del proyecto) brindar los siguientes talleres: la presentación del proyecto Lote 3 y sus beneficios, el sistema actual de agua potable y alcantarillado de Comas, los derechos y deberes de los usuarios, el uso racional del agua y del alcantarillado, hábitos de higiene, el sistema de facturación de SEDAPAL y, finalmente, micromedición y tarifas.

El volante informativo de obra se desarrolló con el objetivo de brindar una información básica sobre el proyecto a la población. Los promotores sociales, a medida que realizaban el reconocimiento de sus zonas de trabajo (tanto de forma geográfica como para la identificación de actores sociales) realizaban la entrega de este medio para ofrecer un primer contenido breve y rápido. Se incluyó información sobre la naturaleza del proyecto, su necesidad y beneficios, la labor del EIS y las formas de contacto para la comunicación con los usuarios. El volante consideró los colores institucionales de SEDAPAL y pasó por siete ediciones antes de su aprobación, la que se dio en coordinación con la supervisión del proyecto. 
Otro producto de comunicación muy utilizado fue el afiche del proyecto. El avance de la obra fue muy rápido. Hubo ocasiones en que las cuadrillas de trabajo llegaban antes de que el equipo social haya informado a la población. Se decidió adelantarse a esa situación y empezar a instalar los afiches en postes de alumbrado y lugares frecuentados (bodegas, mercados y centros educativos). Para la realización del afiche se trabajó con el coordinador del EIS y con un diseñador externo. Al inicio contenía demasiado texto y estaba sobrecargado de elementos. Aunque se mantuvieron varios elementos, se logró una pieza menos saturada y funcional que fue aprobada por la supervisión.

Se incluyó una lista de los beneficios del proyecto (saneamiento de calidad, 24 horas de servicio, menos atoros y aniegos, beneficios económicos, entre otros), siempre tomando en consideración los comentarios de los usuarios vertidos en el estudio de percepciones, a fin de reducir la resistencia que pudiese mostrar la población. También se agregaron imágenes del proyecto que evidenciaban el contacto con la población, así como los trabajos desarrollados para ofrecer una idea de los tipos de intervención.

Pero con el uso se evidenció que tanto el volante informativo y el afiche eran aún insuficientes. Si bien los promotores ofrecieron charlas informativas a las comunidades en sus frentes de trabajo, las demandas de la población por reclamos ante los impactos de la obra (cierre de calles, de cocheras, polvo) eran numerosas y les demandaban gran cantidad de su tiempo. Por ello, requerían una herramienta que permita dar información en mayor detalle. Se elaboró entonces un tríptico informativo. Para su concepción fue necesario un trabajo interno muy detallado, desde utilizar un nombre del proyecto conciso, pero sin que afectara su detalle técnico.

El nombre completo del proyecto era "Optimización del Sistema de Agua Potable y Alcantarillado, Sectorización, Rehabilitación de Redes y Actualización de Catastro - Área de Influencia Planta Huachipa - Área de Drenaje Comas - Chillón - Lima - Lote 3 - Obras de Rehabilitación de Redes Secundarias de Agua Potable y Alcantarillado" (42 palabras en total). Un texto con esa extensión complicaría la comunicación del proyecto. Por ese motivo, para fines del EIS, el nombre del proyecto se redujo a "Lote 3: Rehabilitación de Redes de Agua Potable y Alcantarillado" (10 palabras).

Esta propuesta tuvo que coordinarse con el equipo de ingeniería y con la supervisión, dado que se debía de cumplir con las especificaciones técnicas y los términos de referencia del contrato de la manera más exacta. Una modificación o propuesta inexacta para la difusión del nombre del proyecto podría ser aprovechada por líderes negativos y utilizarla a su favor para levantar a la población con engaños. Entonces, el título se redujo al término que resumía las actividades que tenían un vínculo directo con la población, es decir, la rehabilitación.

Dentro del tríptico se incluyó una descripción del proyecto, incluyendo los esfuerzos que venía realizando SEDAPAL para mejorar los servicios de agua potable y alcantarillado y los beneficios que le generarían para las familias beneficiarias. En este material se detallaba, por ejemplo, los trabajos a realizarse y qué era lo que implicaba la rehabilitación, es decir, el cambio o la instalación adicional de partes del sistema de saneamiento según lo requerido 
por el diagnóstico del proyecto. Rehabilitar no es cambiar todas las tuberías necesariamente, sino sólo las necesarias. Así también, implica instalar algunos componentes para mejorar el rendimiento del sistema, pero no en toda su extensión. También se explicaba a los usuarios que las obras tenían un inicio y un término por calle, de acuerdo con la situación de la red y que todo el proyecto tardaría, en conjunto, algunos meses de trabajo.

Además, se mencionó las afectaciones del proyecto, que eran inevitables, pero que el consorcio trabajaba por hacer que estas incomodidades fueran mínimas. Se detallaba también el nombre del consorcio, su compromiso por hacer un trabajo óptimo, y los números de contacto e incluso la dirección para atender cualquier emergencia o situación anormal. El objetivo del tríptico era transmitir, de forma transparente, el compromiso que tenía la empresa por desarrollar una ejecución de calidad y cercana con el usuario, a fin de reducir esa brecha de desconfianza producto de las experiencias previas.

El EIS también tenía como responsabilidad la capacitación a la población (SEDAPAL, 2011a, p. 32). Así, se desarrollaron rotafolios. Estos materiales de capacitación fueron desarrollados con el fin de que fueran funcionales de forma independiente, es decir, podría ofrecerse una capacitación sin la necesidad de requerir información previa de otra de ellas. Esto facilitaba el trabajo, dado que se escogía el tema más adecuado para cada situación. Por ejemplo, ante los comerciantes de un mercado, el tema de gestión adecuada de residuos sólidos era indispensable. Se había detectado que algunos comerciantes irresponsables utilizaban el desagüe o los buzones de registro de alcantarillado como botadero de plumas y restos de animales. Entonces, se les explicaba las consecuencias de estas acciones y cómo un colapso de los sistemas les afectaría económicamente.

Para el desarrollo de las temáticas se consultaron páginas web, libros, artículos periodísticos y hasta entrevistas con especialistas. La verificación de datos y la opinión de la supervisión siempre fueron necesarias en este proceso.

En el taller 1 ("Presentación del Proyecto Lote 3 y sus beneficios") se describía el proyecto, sus antecedentes, razones, beneficios y también el compromiso de la empresa. El contenido de este taller era la base de lo descrito en el tríptico del proyecto explicado líneas arriba. Fue el taller desarrollado con mayor frecuencia; era el taller base del proyecto. El taller 2 ("El sistema actual del agua potable y alcantarillado de Comas y los deberes y derechos de los usuarios") detallaba la situación de Lima como ciudad desértica y las razones por las que debía considerarse el agua como un recurso valioso en la capital. También explicaba el estado de las redes que a su vez daban razón a la necesidad de la obra.

El taller 3 ("Uso racional del agua y del alcantarillado") trataba cómo debía de utilizarse los sistemas de saneamiento en los domicilios. En el taller 4 ("Hábitos de higiene") se explicaba cómo con el agua y alcantarillado era posible adquirir acciones más eficientes respecto a la limpieza personal y del hogar, y así crear mejores condiciones para nuestro desarrollo: mejorar la salud, el desempeño laboral, nuestros logros personales, etc.

El taller 5 ("El sistema de facturación de SEDAPAL") describía cómo SEDAPAL aplicaba el cálculo de la facturación que aparecía en los recibos de los usuarios. Finalmente, el 
sexto taller se llamaba "Micromedición y tarifas". Explicaba cuál era la diferencia entre la facturación con la tarifa asignada (sin medidor, calculada con promedios) y la que se hacía con medidores, es decir, de forma personalizada. Como ya lo hemos mencionado, los medidores eran un tema polémico y este taller ayudaba a explicar su necesidad.

\section{Conclusiones}

El estudio de percepciones desarrollado permitió conocer no sólo las posiciones a favor o en contra del proyecto, sino especialmente las razones que las sustentaban. Esto facilitó el desarrollo de discursos para promover el proyecto y también neutralizar aquellas opiniones contrarias.

El estudio permitió evidenciar que contratistas previos de SEDAPAL no cumplían con sus actividades, lo que generó un ambiente de desconfianza frente a cualquier otro esfuerzo de trabajos en la zona. Así, se estableció comunicar el compromiso de la empresa frente a esos aspectos.

La investigación abarcó no solamente el análisis de las percepciones sobre el proyecto en sí, sino sobre las obras de agua potable y alcantarillado en general y la micromedición que, aunque no era aplicada por el proyecto, eventualmente la población podría asociarla con el Lote 3 . Es necesario, entonces, considerar en estos estudios no sólo aquellos temas a los que se dedica la empresa sino también otros con los que puedan vincularla y que puedan afectar su relación con los usuarios.

La labor del comunicador social se realizó de manera muy cercana con los promotores sociales, el coordinador de obra, la supervisión e incluso fue necesaria la consulta con fuentes externas y hasta con otros profesionales de mayor experiencia, que permitieron afinar las estrategias.

En el plan de comunicaciones se describió los impactos de obra posibles de ocurrir. Esto permitía justificar las acciones y los mensajes vinculados a esas intervenciones, así como el público primario.

Se planificaron una serie de productos de comunicación que debían responder a diferentes contextos. Esto porque en determinadas zonas los usuarios podrían estar a favor o en contra el proyecto. También porque los usuarios tenían diferentes categorías, esto por los servicios que recibían (agua las 24 horas, agua sólo por horas, con o sin medidor).

El plan de comunicaciones también incluyó las consideraciones del equipo de Seguridad, Salud Ocupacional y Medio Ambiente. Esto porque también era necesario comunicar a los usuarios las medidas a tomar para velar por su integridad física durante los trabajos.

Los dueños de negocios y comerciantes fueron considerados un público especial, dado que sus espacios de trabajo serían claramente afectados. Al abrirse una zanja, el flujo de vehículos, personas y clientes para ellos disminuiría. Por ello, se consideraron acciones especiales para su atención. 
Los mensajes clave desarrollados tenían siempre en consideración al estudio de percepciones. Así, es posible vincular la necesidad de un diagnóstico para un plan de comunicaciones estructurado y afinado a lo que el proyecto requería.

Los mensajes y los productos de comunicación implementados complementaban la estrategia del EIS de estar casi todo el tiempo en campo, permitiendo atender las incidencias rápidamente. Asimismo, los mensajes incorporaban la invocación a los usuarios a hacer un buen uso de las instalaciones sanitarias y, especialmente, a cuidar el recurso hídrico.

Todos los productos de comunicación y de capacitación tomaron en cuenta el estudio de percepciones. En ese sentido, los volantes, afiches, trípticos y rotafolios respondieron a aspectos tales como la desconfianza de los usuarios, las experiencias previas con otros contratistas y su opinión sobre los medidores.

\section{Fuentes consultadas}

Acosta, A. (2010). El buen vivir en el camino. Quito: Fundación Friedrich Ebert, FESILDIS.

Acosta, J. (13 de noviembre de 2014). Entrevista al Licenciado Javier Acosta, ex supervisor del Proyecto Lote 3. Entrevistador: Wilfredo Cornejo Alva.

Alfaro, R. (2006). Otra Brújula. Innovaciones en comunicación y desarrollo. Lima: Asociación de Comunicadores Sociales Calandria.

Aragón, V. (2012). La construcción social del discurso en torno al agua y su contribución a la creación de opinión pública. Murcia: Universidad de Murcia.

Cabrera, L. (2006). Diseño de campaña de comunicación para el desarrollo. Lima: Academia Peruana de Comunicación Organizacional.

Cornejo Alva, W. (2015). Lecciones aprendidas de comunicación para proyectos de rehabilitación de redes de agua y alcantarillado: El caso del lote 3 de Sedapal Comas, Lima (tesis de licenciatura). Universidad Nacional Mayor de San Marcos, Lima, Perú.

Díaz, R. (2011). Desarrollo sustentable. México, DF: McGraw-Hill Educación.

Consorcio Lima Norte, CLN. (2012). Plan de comunicaciones. CLN: Lima.

Fraser, C. \& Restrepo-Estrada, S. (2008). Why Communication? En A. Gumucio-Dagron \& T. Tufte (Comps.), Antología de la comunicación para el cambio social (pp. 792-793). Nueva Jersey: Consorcio de Comunicación para el Cambio Social. 
Frenette, M. \& Reyes, P. (2011). La importancia de las ciencias de la comunicación en las campañas sociales. Derecho a Comunicar. Revista Cientifica de la Asociación Mexicana de Derecho a la Información, 2. Recuperado de https://www. researchgate.net/publication/327989083_La_importancia_de_las_ciencias_de_ la_comunicacion_en_las_campanas_sociales

Fundación Futuro Latinoamericano-FFLA \& Aquafondo (2015). Cuenca interregional Chillón-Rímac y Lurín, Perú. Una experiencia de gobernanza. Sistematización Periodo 2011-2015. Recuperado de https://aquafondo.org.pe/wp-content/ uploads/2016/11/sistematizacion_GA_cuencas-lima.pdf

Instituto Nacional de Estadística e informática, INEI (2017). Provincia de Lima, compendio estadístico 2017. Lima: INEI.

Jamías, J. (2008). Filosofía de la comunicación para el desarrollo. En A. GumucioDagron, \& T. Tufte (Comps.), Antología de la comunicación para el cambio social (pp. 186-190). Nueva Jersey: Consorcio de la Comunicación para el Cambio Social.

Lindon, D. (1977). Marketing político y social. Madrid: TECNIBAN.

Moliner, M. (1998). Marketing social. La gestión de las causas sociales. Madrid: ESIC.

Morel, R. (19 de diciembre de 2012). El reto de las relaciones comunitarias. Recuperado de https://ricardomorel.wordpress.com/2012/12/19/el-reto-de-las-relacionescomunitarias/

Ramiro, L. (2008). La comunicación para el desarrollo en Latinoamérica: una evaluación de cuarenta años. En A. Gumucio-Dagron, \& T. Tufte (Comps.), Antología de la comunicación para el cambio social (p. 614). Nueva Jersey: Consorcio de la Comunicación para el Cambio Social.

Servicio de Agua Potable y Alcantarillado de Lima, SEDAPAL(2010). Encuesta del Nivel de Satisfacción con los Servicios de SEDAPAL. Lima: SEDAPAL. Recuperado de http://www.sedapal.com.pe/c/document_library/get_file?uuid=bf975b66-677f43ba-baa5-7b6d68873f14\&groupId=10154

Servicio de Agua Potable y Alcantarillado de Lima, SEDAPAL(2011a). Especificaciones técnicas generales - Lote 3. Obras de rehabilitación de redes secundarias de agua potable y alcantarillado. Lima: SEDAPAL. Recuperado de http://www. sedapal.com.pe/Contenido/licitaciones/LPI003-2011-JICA-KFW/LPI\%20N\%20 003-2011-JICA-KFW-SEDAPAL/VOLUMEN\%202/ESPECIFICACIONES\%20 TECNICAS/Especificaciones\%20Generales.pdf 
Servicio de Agua Potable y Alcantarillado de Lima, SEDAPAL (2011b). Términos de referencia - Lote 3. Obras de rehabilitación de redes secundarias de agua potable y alcantarillado. Lima: SEDAPAL.

Solano, D. (2009). Responsabilidad social. "º́strategias sostenibles para el desarrollo y la competitividad. Lima: ESAN.

Uribe, C. (2008). Un modelo para armar. Teorías y conceptos de desarrollo. Lima: PUCP.

Vargas, R. (2006). La cultura del agua. Lecciones de la América indígena. Montevideo: UNESCO.

Vizcarra, H. (15 de noviembre de 2014)._Entrevista al Licenciado Humberto Vizcarra, ex coordinador del equipo de intervención social del Proyecto Lote 3. Entrevistador: Wilfredo Cornejo Alva. 